\title{
A Proposed Transition Scheme for the Longitudinal Emittance Control in the Fermilab Booster
}

\author{
Xi Yang, Charles M. Ankenbrandt, James MacLachlan, and Valeri A. Lebedev \\ Fermi National Accelerator Laboratory
}

Box 500, Batavia IL 60510

\begin{abstract}
Instead of applying the $\gamma_{\mathrm{T}}$ jump at the designed value of 1.0, which never can be used in the operation due to the quad steering, the combination of the rf manipulation and a 0.2unit $\gamma_{\mathrm{T}}$ jump can reduce the longitudinal emittance growth nearly $40 \%$ during transition. Especially, a 0.2 -unit $\gamma_{\mathrm{T}}$ jump can help in reducing the rf manipulating voltage from 1000 $\mathrm{kV}$ to $850 \mathrm{kV}$, and makes the transition scheme operationally feasible.
\end{abstract}

\section{Introduction}

The transition-jump system (TJS) has been installed in the Fermilab Booster since 1987, for the purpose of reducing the deleterious effects of a high intensity beam passing through transition via reducing the time that the beam spends near the transition energy.[1,2] Because of the quad steering, which is caused by the beam not being well centered through all the $\gamma_{\mathrm{T}}$ quads, the TJS has never been used in the operation.

A program, which uses the difference in the closed orbits when $\gamma_{\mathrm{T}}$ quads are on and off and calculates the offsets of the beam relative to $\gamma_{T}$ quads, has been successfully developed and applied to find the optimal position for centering the beam through all the $\gamma_{\mathrm{T}}$ quads.[3] Also, a radial orbit offset (ROF) has been experimentally applied to move the beam onto the optimal radial position during the $\gamma_{\mathrm{T}}$ waveform, which covers transition crossing (TC) to $5 \mathrm{~ms}$ after transition.[3] However, any vertical offset requires 
the reposition of the beam relative to the $\gamma_{\mathrm{T}}$ quad either by applying a local three-bump to the beam or by moving the $\gamma_{\mathrm{T}}$ quad. Furthermore, the cogging, which has been implemented for slip stacking two Booster batches into one Main Injector high intensity batch, $[4,5]$ is operationally used to synchronize the beam notch in Booster and the trigger of the Booster-to-Main Injector transfer via moving the beam radically right after transition. As a result of the cogging, the radial position of the beam couldn't be fixed for the purpose of commissioning the TJS any more.

\section{Design Considerations}

Since the smaller the $\gamma_{T}$ jump $\left(\Delta \gamma_{T}\right)$ is, the lower the magnetic field of the $\gamma_{T}$ quads is, and the less the quad steering is. It's important for us to develop a procedure of commissioning the TJS, with a small $\Delta \gamma_{\mathrm{T}}$ for the purpose of minimizing the quad steering. However, the time that the beam spends near the transition energy increases with the decrease of $\Delta \gamma_{\mathrm{T}}$; and most of the deleterious effects occur right after TC due to the following: $1^{\text {st }}$, longitudinal space charge (SC) forces defocus the beam bunch before transition and focus the beam bunch after transition;[6] $2^{\text {nd }}$, since the bunch length reaches the minimum after transition, and at the same time, SC forces reach the maximum, the strongest repulsive $\mathrm{SC}$ forces lead to the particles in the beam approaching each other longitudinally and excite the negative mass instability;[6] $3^{\text {rd }}$, and above the non-adiabatic TC till the bunch length reaches the minimum, there is a mismatch between the beam bunch and the rf bucket, and such a mismatch excites the bunch length oscillation and causes the longitudinal emittance (LE) growth.

Considering that in the longitudinal phase space synchronous motions are frozen during TC, and any change in the momentum coordinate is almost decoupled from the phase coordinate, rf manipulations have been implemented during TC.[7] One can increase the momentum spread $(\Delta p)$ right before TC via increasing the rf accelerating voltage $\left(V_{R F}\right)$ without significantly varying the bunch length, and such an increase in $\Delta p$ before TC contributes to the defocusing force after TC, and keeps the minimum bunch length from getting too short.

Compared to the designed $\Delta \gamma_{\mathrm{T}}=1.0, \Delta \gamma_{\mathrm{T}}$ that can be used in the operation is about 0.2 . Therefore, instead of applying the TJS to increase the TC rate, one can apply 
the TJS with $\Delta \gamma_{\mathrm{T}}=0.2$ to minimize the deleterious effects right after TC in conjunction with the rf manipulation. This can be done by triggering the TJS at the same time with the normal TC gate, and the 0.2 -unit $\Delta \gamma_{\mathrm{T}}$ right after transition can significantly reduce the time that the beam spends in the non-adiabatic transition region and the chromatic nonlinear mismatch region.[8] Since in the non-adiabatic transition region, the effect of the non-linear dependence of the slip factor on the momentum deviation becomes important, the amount of the $\Delta p$ increase via the rf manipulation should be re-adjusted based upon the 0.2 -unit $\Delta \gamma_{\mathrm{T}}$.

\section{Numerical Simulation}

ESME simulations are used to search for the optimal setting for the combination of both the rf manipulation and the 0.2-unit $\Delta \gamma_{\mathrm{T}}$ during TC.

Since the $V_{R F}$ curve already has been optimized for the purpose of minimizing LE and $\Delta p$ of the $8-\mathrm{GeV}$ proton beam,[7] it can be directly used in ESME simulations. Except that the amplitude of these two rf manipulating pulses, one right before transition and one right after transition, should be re-adjusted according to the 0.2-unit $\Delta \gamma_{\mathrm{T}}$.

ESME simulations are done for the extracted beam intensity of $4.0 \times 10^{12}$, at the configuration of combining both the rf manipulation and the 0.2-unit $\Delta \gamma_{\mathrm{T}}$ during TC. Here, the trigger of the $\gamma_{T}$ waveform is chosen to be at the normal transition time, about $16.9 \mathrm{~ms}$ in the cycle. The $\gamma_{\mathrm{T}}$ waveform is shown in Fig. 1(a). The $1^{\text {st }} \mathrm{rf}$ pulse is optimized, and the result is: $16.30 \mathrm{~ms}$ to $16.35 \mathrm{~ms}, V_{R F}$ linearly increases from $700 \mathrm{kV}$ to $850 \mathrm{kV} ; 16.35 \mathrm{~ms}$ to $16.90 \mathrm{~ms}, V_{R F}$ stays at the constant value of $850 \mathrm{kV}$; and, $16.90 \mathrm{~ms}$ to $16.95 \mathrm{~ms}, V_{R F}$ linearly decreases from $850 \mathrm{kV}$ to $700 \mathrm{kV}$. And the $2^{\text {nd }} \mathrm{rf}$ pulse is optimized, and the result is: $17.30 \mathrm{~ms}$ to $17.31 \mathrm{~ms}, V_{R F}$ linearly increases from $700 \mathrm{kV}$ to $850 \mathrm{kV} ; 17.31 \mathrm{~ms}$ to $17.39 \mathrm{~ms}, V_{R F}$ stays at the constant value of $850 \mathrm{kV}$; and, $17.39 \mathrm{~ms}$ to $17.40 \mathrm{~ms}, V_{R F}$ linearly decreases from $850 \mathrm{kV}$ to $700 \mathrm{kV}$. $V_{R F} v s$. time in a cycle is shown in Fig. 1(b). EPSILON vs. time is shown in Fig. 1(c). For a Gaussian distribution, the area containing $95 \%$ of the beam is six time EPSILON.

The phase and momentum projections at 8-GeV are shown as Figs. 1(d) and 1(e) respectively. $\Delta p$ in rms and LE with $95 \%$ of the beam at $8-\mathrm{GeV}$ are $2.98 \mathrm{MeV}$ and $0.0538 \mathrm{eV} \cdot \mathrm{sec}$, and compared to the situation without both the rf manipulation at TC (two 
rf pulses $)$ and the $\gamma_{\mathrm{T}}$ jump, there is a $\left.39 \%(\approx(0.088-0.0538) / 0.088)\right)$ reduction in LE and a $5.6 \%(\approx(3.156-2.98) / 3.156))$ reduction in $\Delta p$.

\section{Conclusions}

Since the cogging requires the beam to be moved radically right after transition, only a small $\Delta \gamma_{\mathrm{T}}$ could be feasible in the operation. Instead of applying the TJS at the designed value of $\Delta \gamma_{\mathrm{T}}=1.0$ to make TC faster, the TJS is triggered at the normal transition time for the purpose of reducing the time that the beam spends in the non-adiabatic region after transition. With the combination of both the $\mathrm{rf}$ manipulation and the $\Delta \gamma_{\mathrm{T}}=0.2$, at the extracted beam intensity of $4.0 \times 10^{12}$ protons, LE of $0.0538 \mathrm{eV} \cdot \mathrm{sec}$ and $\Delta p$ of $2.98 \mathrm{MeV}$ at $8-\mathrm{GeV}$ can be achieved at the manipulating $V_{R F}$ of $850 \mathrm{kV}$. Compared to the situation with the rf manipulation only,[7] the 0.2-unit $\Delta \gamma_{\mathrm{T}}$ can help in reducing the manipulating $V_{R F}$ from $1000 \mathrm{kV}$ to $850 \mathrm{kV}$ and with several percent more LE reduction. Especially, the $850-\mathrm{kV}$ manipulating $V_{R F}$ is much easier to be achieved in the operation.

\section{References:}

[1] L. C. Teng, Compensation of Space-charge Mismatch at Transition of Booster Using the Transition-jump Method, FERMLAB-FN-207.

[2] W. Merz, C. Ankenbrandt, and K. Koepke, Transition Jump System for the Fermilab Booster, FERMILAB-TM-1473.

[3] X. Yang, etc., "Study Report of the Booster Transition Jump System", FERMILABTM-2287-AD.

[4] K. Koba, etc., "SLIP STACKING EXPERIMENTS AT FERMILAB MAIN INJECTOR”, FERMILAB-CONF-03-107.

[5] K. Koba and J. Steimel, "SLIP STACKING”, FERMILAB-CONF-02-205.

[6] D. A. Edwards and M. J. Syphers, An Introduction to the Physics of High Energy Accelerators, 1993, John Wiley \& Sons, Inc.

[7] X. Yang, etc., "Reducing the Longitudinal Emittance of the 8-GeV Beam via the rf Manipulation in a Booster Cycle”, FERMILAB-FN-0769-AD, submitted.

[8] J. Wei, "Longitudinal Dynamics of the Non-Adiabatic Regime on AlternatingGradient Synchrotrons", Ph.D thesis, State University of New York, 1990. 


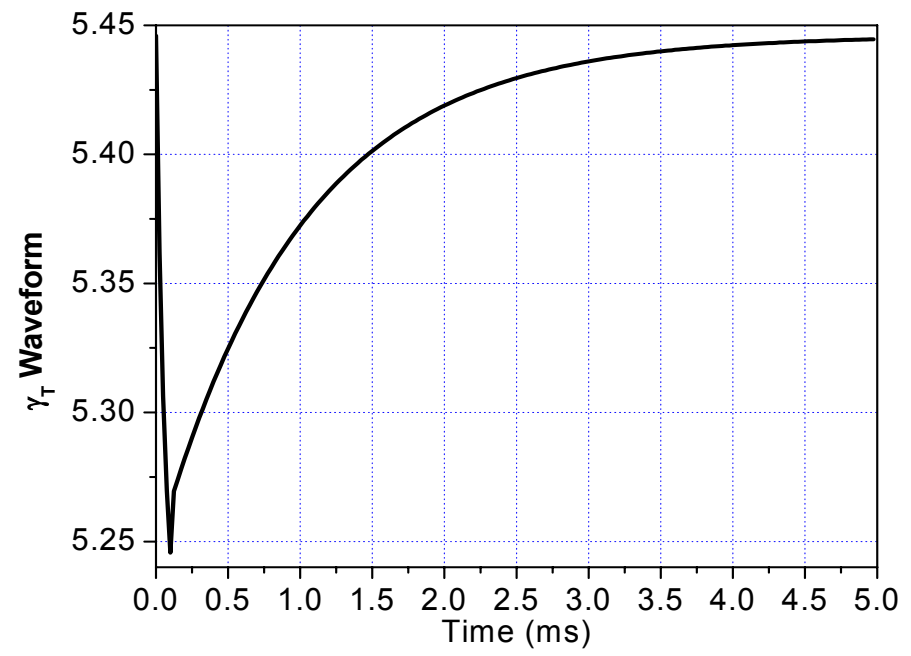

Fig. 1(a)

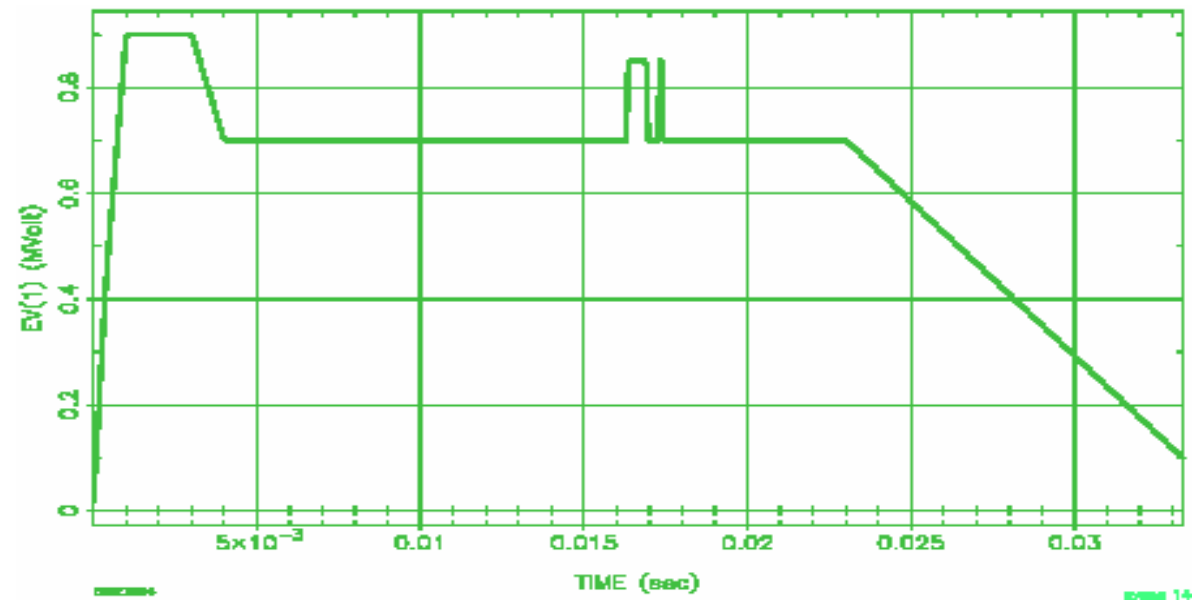

Fig. 1(b)

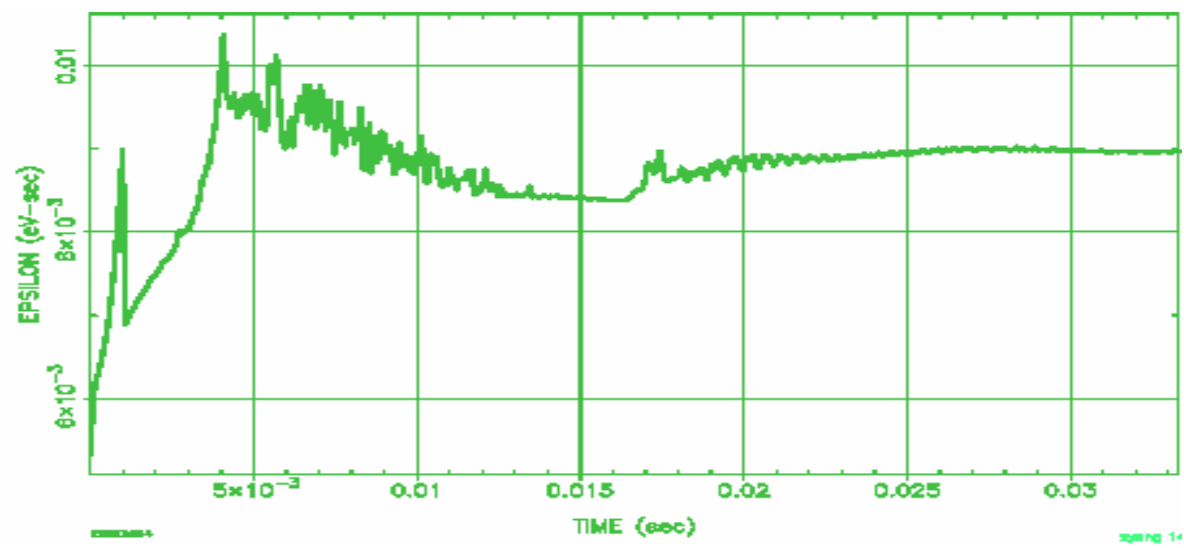

Fig. 1(c) 


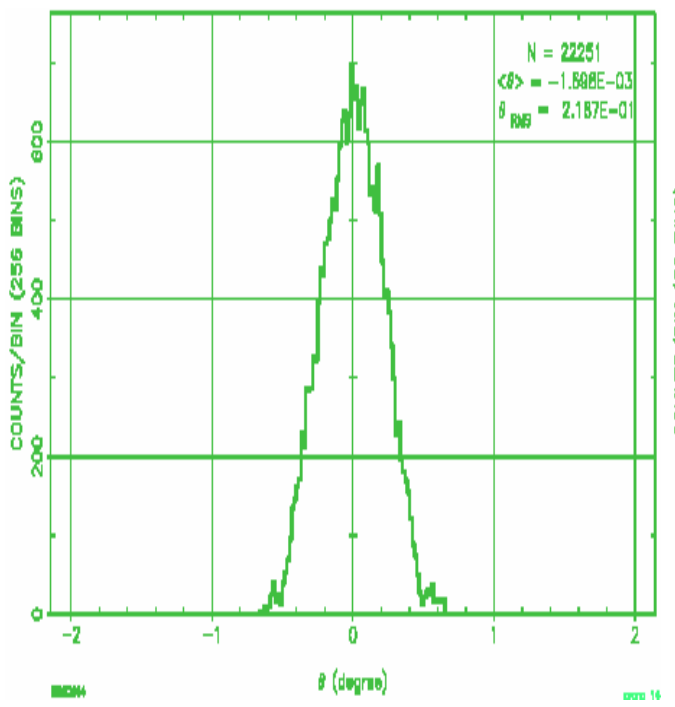

Fig. 1(d)

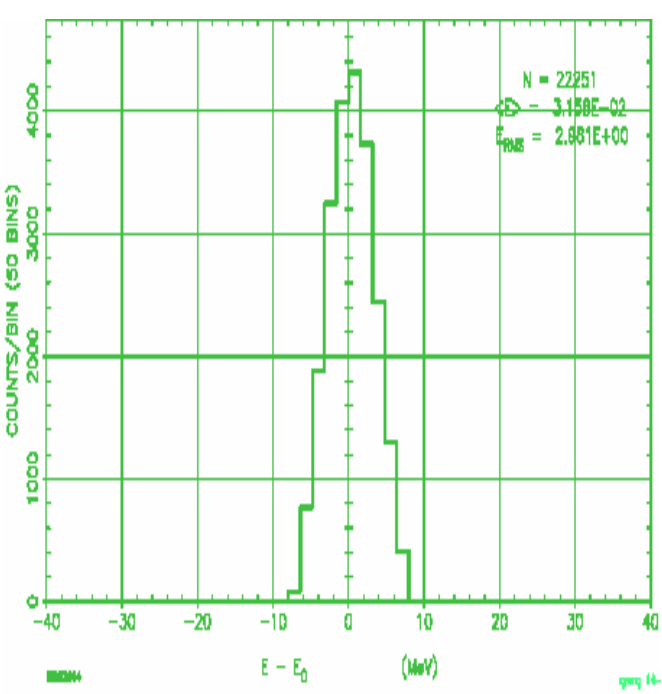

Fig. 1(e)

Fig. 1(a) $\gamma_{\mathrm{T}}$ waveform at the maximum $\Delta \gamma_{\mathrm{T}}$ of 0.2 .

Fig. 1(b) $V_{R F} v s$. time in a cycle.

Fig. 1(c) with both the rf manipulation and $\Delta \gamma_{\mathrm{T}}=0.2$, at the extracted beam intensity of $4.0 \times 10^{12}$ protons, EPSILON vs. time.

Fig. 1(d) the phase projection at $8-\mathrm{GeV}$.

Fig. 1(e) the momentum projection at $8-\mathrm{GeV}$. 